Proceedings of SALT 20: 387-405, 2010

\title{
A Montagovian treatment of modal subordination
}

\author{
Nicholas Asher \\ asher@irit.fr \\ IRIT/CNRS
}

\author{
Sylvain Pogodalla \\ Sylvain.Pogodalla@inria.fr \\ LORIA/INRIA Nancy - Grand Est
}

\begin{abstract}
Epistemic modality involves complex contextual dependencies that linguists have studied (Roberts 1989; Veltman 1996). We show that they have a natural treatment within a continuation style semantics.
\end{abstract}

Keywords: semantics, modal subordination, continuations, discourse, dynamics, logic, type-theory

\section{Introduction/Abstract}

Epistemic modality involves contextual dependencies in discourse of at least two kinds (Roberts 1989; Veltman 1996). We characterize them with the slogans:

I Epistemic modal facts are asymmetrically dependent on non modal facts.

II Some modal facts are dependent on other modal facts.

We show that they have a natural treatment within a continuation style semantics.

The first contextual dependency is on display in Veltman's (1996) examples:

(1) It might be sunny. But it's not sunny

(2) \# It's not sunny. But it might (for all I know) be sunny.

For Veltman, there is a subtle but unmistakable difference between (1) and (2). The first involves a smooth discourse update, whereas the second does not. The non modal facts established in prior discourse constrain the set of epistemic possibilities: if the discourse context contains a non modal fact that $p$, it does not felicitously admit a continuation with the epistemic possibility that $\neg p$. The converse, however, does not hold: if the discourse context contains a modal fact that $p$, it may admit a felicitous continuation that establishes the non modal fact that $p$.

The phenomenon known as modal subordination (MS) exemplifies the second dependency of epistemic modalities. MS involves anaphoric links between pronouns and their antecedents across modalities like those in (3) and (4) from Roberts 1989. Standard versions of dynamic semantics (Kamp 1981) do not predict these links. 
(3) A wolf might enter. It would growl.

(4) A wolf might enter. \#It will growl.

(5) A wolf enters. ?It would growl.

Previous accounts of dependencies (I) and (II) have exploited DRT or other dynamic frameworks (Roberts 1989; Veltman 1996; Frank \& Kamp 1997; Stone $\&$ Hardt 1997). We extend the Montagovian continuation semantics of Philippe de Groote (2006), to provide a modular treatment of both MS and V. We embed dynamic modal logics within a classical setting, where quantifiers, modal operators and variables receive their standard interpretation and assignments and discourse referents are not semantic objects in the models, as they are in most other analyses.

\section{An introduction to continuation semantics}

Most versions of dynamic semantics (DS) in linguistics make heavy use of assignment functions as semantic objects. While this may not be apparent to the casual reader of early work like Kamp 1981, a compositional semantics for DRT, DPL (Groenendijk \& Stokhof 1991) or more recent developments (Brasoeavnu 2008) leads almost inevitably to the introduction of "odd" types whose inhabitants are variables, assignments or other "representational" elements. Dynamic Intensional Logic (DIL) (Groenendijk \& Stokhof 1996; Dekker 1999), following Janssen's (1983) work on programming languages, has the virtue of making central the semantic status of assignment functions, as they are the points of evaluation in that model theory. But almost all of extant versions of DS include assignment functions as parts of semantic values. The exploitation of assignment functions as semantic objects plays a crucial role in many dynamic semantics approaches to modal subordination and intensionality (Frank \& Kamp 1997; Asher \& McCready 2007). This engenders subtle differences in the underlying logic, clouding the logical status of discourse referents in a "top" DRS by making them appear ambiguous between existentially bound variables and free ones (cf. Fernando 1994). It also leads to problems of destructive assignment in DPL and with variable clash in DRT or versions of DPL that use partial assignment functions. It is difficult to avoid these problems in a purely compositional environment, leading to cumbersome systems.

Continuation style semantics (CS) developed by computer scientists in the 70s and introduced into linguistics by Barker (2004), Shan \& Barker (2006), Philippe de Groote (2006), and Bernardi \& Moortgat (2010) avoid these problems. CS provides a more abstract setting for dynamic logics, abstracting away from assignments that are essential to the formulations of DIL, DPL and DRT. CS, like DS, models the dynamic meaning of a natural language expression as a transition between a left 
context and a right one. But in CS right contexts are explicitly introduced and are defined in terms of left contexts and sentence denotations. CS exploits the structure of a monad in category theory (Moggi 1991), which specifies the parameters needed to provide a CS: the first is to specify what a left context is; the second is to provide a "binder" rule, which tells us how to combine the semantics of a text with that of subsequent sentence; the third is to specify the lexical entries for expressions. CS thus refocuses semanticists' attention on specifying appropriate lexical entries and discourse contexts. CS permits a wide variety of choices as to what left contexts, binder rules and lexical entries are, a liberalism we exploit below.

As an example, consider Philippe de Groote's (2006) CS. de Groote exploits Montague's homomorphic interpretation of syntactic types and structures into semantic types and terms. But Philippe de Groote (2006) changes Montague's interpretation $\llbracket s \rrbracket$ of the sentence type $s$ from $t$ to: $\Omega \triangleq \partial \rightarrow(\gamma \rightarrow t) \rightarrow t$, where $\gamma$ is the type of the left context or discourse context already given and $\gamma \rightarrow t$ is the type of the right context or discourse "to come"-its continuation. If this sentence introduces a new discourse entity $x$, given an environment $i$ (as input) and a continuation $k$ as parameter, it can provide $(x:: i)$ (with $\cdot:: \cdot$ a list constructor of type $e \rightarrow \gamma \rightarrow \gamma)$ as parameter to $k$, making the value of $x$ available for $k$.

Other types have standard interpretations. Where $\llbracket X \rrbracket$ stands for the $\lambda$-term or meaning of $X, \llbracket n p \rrbracket=(e \rightarrow \llbracket s \rrbracket) \rightarrow \llbracket s \rrbracket$ and $\llbracket n \rrbracket=e \rightarrow \llbracket s \rrbracket$. Pronouns have the following interpretation: $\llbracket i t \rrbracket=\lambda P . \lambda i k . P(\operatorname{sel} i) i k$, where $i: \gamma, k: \gamma \rightarrow t$, and where sel $i$ is a function that selects a suitable discourse antecedent inside $i$.

A CS like Philippe de Groote's (2006)'s must say how a text $T$ combines with a sentence to its right. This is the CS binder issue, which is also an essential part of all dynamical systems-it is the DRS update operation of DRT or relational composition of sentence contents in DPL. Here is the basic binder equation for de Groote's system and ours:

$$
\llbracket T . S \rrbracket=\lambda i . \lambda k \cdot \llbracket T \rrbracket i\left(\lambda i^{\prime} . \llbracket S \rrbracket i^{\prime} k\right)
$$

That is, the text to date $T$ takes the meaning of $S$ as its right context, or rather the meaning of $S$ suitably applied and abstracted so that it can be of $t$ type. A quick type

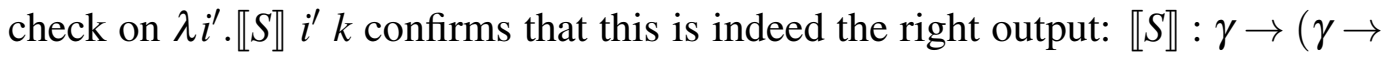
$t) \rightarrow t$ so $\lambda i^{\prime} . \llbracket S \rrbracket i^{\prime} k: \gamma \rightarrow t$.

Let's look at a sample discourse to see how the theory works.

A man is sleeping. He is snoring.

Suitable lexical entries provide a completely classical interpretation of these two sentences except that they have both a left and right context and that the second sentence fills in the right context of the first. The entries also make the existential 
determiner introduce an individual into the right context - a witness that will be "selected" by the pronoun. With this in mind, the interpretations of the first and second sentences are:

$\lambda i . \lambda k . \exists x .(\operatorname{man} x) \wedge(\operatorname{sleeping} x) \wedge(k(x:: i)) \quad \lambda i . \lambda k .(\operatorname{snoring}(\operatorname{sel} i)) \wedge(k i)$

Using the binder rule in (6), these last two $\lambda$ terms yield a meaning for (7) :

$$
\begin{aligned}
& \lambda i k \cdot([\lambda i k . \exists x .(\operatorname{man} x) \wedge(\text { sleeping } x) \wedge k(x:: i)] i) \\
& \left(\lambda i^{\prime} .(\lambda i k .(\text { snoring }(\operatorname{sel} i)) \wedge(k i)) i^{\prime} k\right) \\
& \rightarrow_{\beta} \lambda i k \cdot[\lambda k \cdot \exists x \cdot(\operatorname{man} x) \wedge(\text { sleeping } x) \wedge(k(x:: i))] \\
& \left.\left(\lambda i^{\prime} \text {.(snoring }\left(\operatorname{sel} i^{\prime}\right)\right) \wedge\left(k i^{\prime}\right)\right) \\
& \rightarrow_{\beta} \lambda i k \cdot\left[\exists x .(\operatorname{man} x) \wedge(\text { sleeping } x) \wedge\left(\left(\lambda i^{\prime} .\left(\text { snoring }\left(\operatorname{sel} i^{\prime}\right)\right) \wedge\left(k i^{\prime}\right)\right)(x:: i)\right)\right] \\
& \rightarrow_{\beta} \lambda i k \cdot[\exists x \cdot(\operatorname{man} x) \wedge(\operatorname{sleeping} x) \wedge((\operatorname{snoring}(\operatorname{sel}(x:: i)) \wedge(k(x:: i))))]
\end{aligned}
$$

In selecting the bound variable $x$, the semantic value of the anaphoric pronoun falls within the scope of the original existential quantifier, reducing anaphoric binding to quantificational binding.

We briefly compare de Groote's CS to DIL. ${ }^{1}$ The points of evaluation in models for DIL are not worlds but assignments of values to variables. Sentences have the type: $(s \rightarrow t) \rightarrow t$, where $s$ is the type of states (assignments). Crucial to DIL's account of intersentential anaphora are the modalities, $\langle x / d\rangle$, where $d$ identifies an assignment. $\langle x / d\rangle \phi$ evaluates $\phi$ relative to an assignment in which the value of $d=$ the value of $x$ in the current state. In DIL, quantification over $x$ in $\langle x / d\rangle \phi$ provides dynamic binding, and as Dekker (1999) emphasizes:

$$
\lambda p \cdot \exists x \cdot\langle x / d\rangle^{\vee} p\left[^{\wedge} U d\right] \rightarrow_{\beta} \exists x . U x
$$

Like CS, DIL uses a binder rule to build text meanings:

$$
\llbracket T \rrbracket \cdot \llbracket S \rrbracket=\lambda p\left(\llbracket T \rrbracket\left(\wedge^{\wedge} \llbracket S \rrbracket(p)\right)\right.
$$

Returning to (7), DIL provides the following logical forms for the two sentences.

$$
\left.\left.\lambda p . \exists x\langle x / d\rangle .(\operatorname{man} x) \wedge(\text { sleeping } x) \wedge^{\vee} p\right) \quad \lambda p \text {.snoring }(d) \wedge^{\vee} p\right)
$$

Exploiting (8), the translations (10), together with (9), now yield for (7):

$$
\left.\lambda p . \exists x .(\operatorname{man} x) \wedge(\text { sleeping } x) \wedge \text { snoring }(d) \wedge^{\vee} p\right)
$$

1 DIL's Montagovian nature facilitates the comparison, but we could have illustrated our points with other versions of DS. 
A Montagovian treatment of modal subordination

The DIL derivation capitalizes on the reduction properties of state switcher $\langle x / d\rangle$ and crucially depends on DIL's unorthodox "worlds", which are assignments. This is quite different from Philippe de Groote's CS, whose model theory and logic are completely classical. The left context list structure builds in effect assignment functions internally, via the interpretation of update operator :: and captures the structure of an assignment function without postulating any as basic objects in the model theory. ${ }^{2}$ Notice that this simplification comes about simply by a "distribution of labor": the binder rule in Philippe de Groote 2006 is more complex than that of DIL as are the lexical entries. But the payoff is a logical framework that is just that of standard logic.

\section{Modality}

\subsection{Veltman's test}

In this section, we address our first slogan, which encapsulates the context dependency of epistemic modality upon the non modal facts taken to be common ground in the discourse. This intuition has proved useful in a number of areas of semantics and epistemology. For instance, it yields a simple formulation of the Ramsey test semantics for conditionals. It was Frank Veltman who brought this feature of modals into contemporary discussions of epistemic modality with the examples we repeat below:

(12) It might be sunny. But it's not sunny.

(13) \# It's not sunny. But it might (for all I know) be sunny.

Veltman observes that (12) involves a smooth discourse update, whereas (13) does not. To account for his observation, he builds a semantics for a propositional modal language in which all sentences define an update function on an information state, which is modelled as a set of worlds or epistemic possibilities. The information state evolves as discourse proceeds. All non modal sentences $\phi$ have a particularly simple update $[\phi]:(s[\phi])=\{w \in s: w \in\|\phi\|\}$. Modal sentences, however, involve tests on the information state. For a sentence of the form $\diamond \phi$, we have: ${ }^{3}$

$$
s[\diamond \phi]=s \text { iff } \exists w \in s w \in\|\phi\|
$$

2 The semantics would work just as well if, instead of lists, we had used sets of objects as left contexts.

3 We define a Ramsey test semantics for conditionals, according to which a conditional $\phi \Rightarrow \psi$ is true just in case updating one's information state with $\phi$ and then with $\psi$ is equivalent to updating just with $\phi$, as follows for a propositional language:.

$$
s[\phi \Rightarrow \psi]=s \text { iff } s[\phi][\psi]=s[\phi]
$$


Veltman's dynamic semantics is of a very different style from DRT or DPL, where sentences define dynamic transitions over assignment functions or world assignment function pairs. Philippe de Groote's CS picks a notion of left context appropriate for modelling DRT or DPL; but to recreate the sort of test behavior by modals in Veltman's semantics, we need to have a different notion of left context. As we said in the previous section, however, one of the principal virtues of a continuation style semantics is that a left context can in principle be any sort of data structure without changing the basic set up of the semantics; this is a feature that we exploit here in modelling Veltman's observations within CS. We will take a left context to be a theory or collection of sentences; this theory models the information state and will evolve as discourse proceeds, just as the information state does for Veltman. We will then formulate the semantics of modals using a notion of a test on such a state to capture the behavior that Veltman noticed.

\subsubsection{Veltman modalities within continuation semantics}

In this section we provide a Veltman-inspired CS for a propositional modal language $\mathfrak{L}$, containing propositional variables, $\neg, \wedge$, and $\diamond$. To incorporate the notion of a test, modals of the form $\diamond \phi$ permit a continuation when the modal context $i_{1}$ is such that $i_{1} \cap\|\phi\| \neq 0$. This is a presupposition that the incoming context must meet. If the presupposition is satisfied, then the update is defined and discourse processing can proceed. If the discourse context does not satisfy the presupposition, there is no accommodation and no further updating is possible.

Veltman's idea of a test treats the semantic value of a formula as something other than a simple truth value. Thus, our type of proposition must reflect this; it will no longer be $t$, as in extensional versions of Montague grammar, but rather some intensional type, which we will call here prop. We consider left contexts to be a theory, of type Theory, giving the content of the discourse context (this could be a single formula or a set of formulas). Just as in Philippe de Groote 2006, we also need the :: operator that updates the left context within information from the formula under consideration. The basic types of our variables $i$ (for left contexts), $k$ (for right contexts) and :: (for our update function) are:

$$
i: \gamma \triangleq_{\text {Theory }} \quad k: \text { Theory }_{\rightarrow} \rightarrow \quad:: \text { prop } \rightarrow \text { Theory } \rightarrow \text { Theory }
$$

We also have an exception Halt that when raised stops the evaluation, and a TEST operation that we define presently. We now define the dynamic variants of the propositional connectives and modal operator, with a canonical injection from the static to the dynamic case. The basic $\lambda$-terms of this language are:

- for an atomic static formulas $p$ of type $t: \lambda i k \cdot p \wedge(k(p:: i))$; 
A Montagovian treatment of modal subordination

- for $P$ a dynamic proposition (of type $\Omega \triangleq{ }^{\Delta} \gamma \rightarrow(\gamma \rightarrow t) \rightarrow t$ )), we define (with $\mathbb{T} \triangleq \lambda i$. $\top$ the trivial continuation):

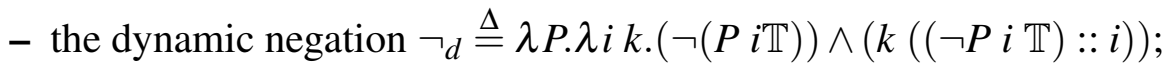

- the dynamic conjunction: $\wedge_{d} \triangleq \lambda P Q . \lambda i k . P i\left(\lambda i^{\prime} . Q i^{\prime} k\right)$;

- the dynamic modality $\diamond_{d} \triangleq \lambda P . \lambda i k$. (TEST $\left.P\right) i\left(\lambda i^{\prime} .\left(k i^{\prime}\right) \wedge\left(\diamond\left(P i^{\prime} \mathbb{T}\right)\right)\right)$ where $\diamond$ is the classic static modality.

To define TEST, we need to evaluate the consistency of the claim under the scope of the modal with the incoming context. We use an evaluation function EVAL from propositions to truth values, which we define in terms of consistency or general satisfiability (relative to any model). ${ }^{4}$

$$
\text { TEST } P=\lambda i k . \text { if }(\text { EVAL } P i \mathbb{T}) \text { then }(k i) \text { else (raise Halt) }
$$

$\nabla_{d}$ yields a continuation iff there is at least one world that verifies all the information in the discourse context together with the content under the scope of the modal. If not it raises an exception Halt, whose effect is to stop the evaluation. This captures Veltman's intuition that there is no possible continuation in this case. This use of exceptions resembles de Groote \& Lebedeva's (2010) use of exceptions for presupposition accomodation. This is no surprise; Veltman's might exhibits presuppositional behavior, but with no possibility of accommodation.

This set up makes the same predictions that Veltman's semantics does for simple modal sentences, assuming the standard combination rule (which actually is $\wedge_{d}$ ). We illustrate with (12). Beginning with the empty input context empty and updating with 'It might be sunny' $\left(\diamond_{d} S\right.$ with $S=\lambda i k$.sunny $\wedge(k$ (sunny $\left.:: i)\right)$ ) we get a continuation because it is consistent to assume $S$ (and indeed any propositional letter), that is EVAL $S$ empty $\mathbb{T}=\top$.

$$
\begin{aligned}
\left(\diamond_{d} S\right) \text { empty } & \rightarrow_{\beta} \lambda k \cdot(\text { TEST } S) \text { empty }\left(\lambda i^{\prime} \cdot\left(k i^{\prime}\right) \wedge\left(\diamond\left(S i^{\prime} \mathbb{T}\right)\right)\right) \\
& \rightarrow \beta k \text { empty }
\end{aligned}
$$

Integrating this with 'not sunny' or $\lambda i k .(\neg$ sunny $) \wedge(k((\neg$ sunny $):: i))$ using (6) will then restrict future continuations to having a discourse context in which it is not

4 We could also define EVAL relative to a particular model as does Veltman. But if the fixed model is somehow deficient (doesn't for instance contain enough epistemic possibilities), the Test procedure may produce funny results. While one can stipulate a full model in cases where canonical model constructions are possible, this is not always possible. Our conception of EVAL does not have this limitation. 
sunny as a left context. But reversing the order of these sentences gives us a different result. Updating first with $\neg_{d} S$, gives us a discourse context such that attempting to update with $\vartheta_{d} S$ will lead the TEST to raise Halt because the current theory to be tested will contain both $\neg$ sunny and sunny which makes it unsatisfiable. This yields no continuations, and no further informative updates are possible. We thus predict that the discourse is malformed. Veltman's account of update to test and its consequence are straightforwardly captured in this CS.

\subsection{Modal subordination}

We turn now to our CS analysis of the interdependence of modal facts and MS. Treating modality as a standard Kripke operator in DRT makes bad predictions:

(17) A wolf might walk in. It would growl.

(18) A wolf might walk in. It *will growl

(19) A wolf is outside. He might growl.

DRT predicts (18) to be bad and (19) to be good, as intuitions warrant. But it also predicts (17) is bad. A straightforward analysis of modality in DRT produces two subDRSs in the main DRS, making the discourse referents introduced under the scope of one modal inaccessible to anaphoric conditions in the main DRS, or to conditions inside the scope of the second model. This yields bad results.

To address this problem, Roberts (1989) treats $\square$ and $\diamond$ as generalized quantifiers whose unspecified restrictors can be accommodated in the context. $\square \phi$ is in fact: $\square(?, \phi)$, with ? a modal base to be filled in the context using a proposition within the scope of an appropriate modal.

In Roberts 1989, (17) yields the DRS (using the standard linear notation):

$$
\diamond(?,[x \mid \text { wolf } x \ldots]) \wedge \square([[x \mid x(\text { wolf } x \ldots],[\mid \text { growl } x]])
$$

Roberts's account doesn't specify a direct anaphoric link between the pronoun and the linguistically given antecedent in its own modal context. The discourse referent $x$ introduced by the first sentence remains inaccessible to the pronoun introduced in the second. But there is an inferential link added between the linguistic antecedent in the first sentence and the pronoun; the second sentence in fact quantifies over all elements in the appropriate domain that have the property specified by the first sentence, and thus we can infer that whatever the witness for $x$ in the first DRS, it will growl in every relevant epistemic possibility.

Geurts (1996) and Frank (1996) criticize Roberts's account as being too unconstrained. They postulate a direct anaphoric link at the level of sentence contents between the restrictor of the second modality and the material in the nuclear scope 
A Montagovian treatment of modal subordination

of the first modality. The value assigned to $x$ in the first sentence is linked to its interpretation of the second sentence in Frank \& Kamp 1997 because the assignments to $x$ are encoded in the semantic value (the dynamic intension) of the material under the scope of the $\diamond$ and hence in the restrictor of the would modality. This strategy shows that the discourse referent $x$ is not functioning as an existentially quantified variable.

But in Frank \& Kamp 1997 the would modality disappears into the conditional $\Rightarrow$. They generate a very weak modal logic, and their account is still too unconstrained (Asher \& McCready 2007). Finally, (21) shows that a straightforward interpretation of the modal structures above leads to trouble.

(21) A wolf might walk in. He would growl. A second wolf might then walk in, but he wouldn't growl.

If the would modality is understood as a simple universal quantification over all wolves that walk in, this discourse is predicted to be contradictory, but it isn't. This is a clearly problem for Roberts' account. Frank and Kamp interpret the would modality relative to all those normal epistemic possibilities satisfying the antecedent, and this may permit them to avoid the prediction. But this wiggle room also further weakens the account's predictions-for instance, among those epistemic possibilities validating (17), there may be epistemic possibilities where the wolf doesn't growl, contrary to intuitions.

\subsubsection{The modal context}

We first show how to make a modal context accessible to further modalized continuations. We interpret sentences and discourse as described in (22), together with the sentence combination rule of (23). Table 1 then introduces the standard dynamic lexicon we use. This lexicon uses the following notational convention: syntactic constituents (basically trees in this paper) are represented as $c_{\text {word }}$, to be read as word. A sentence such as a wolf might enter is represented by its syntactic tree $c_{\text {might }} c_{\text {enter }}\left(c_{a} c_{\text {wolf }}\right)$. The latter shows the application rules to be considered in compositionally computing the semantic representation.

$$
\begin{aligned}
\llbracket s \rrbracket & =\gamma \rightarrow(\gamma \rightarrow t) \rightarrow t & & \triangleq \\
\llbracket S_{1} \cdot S_{2} \rrbracket & =\lambda i k \cdot \llbracket S_{1} \rrbracket i\left(\lambda i^{\prime} . S_{2} i^{\prime} k\right) & & : \Omega
\end{aligned}
$$

In order to deal with modalities, we introduce the interpretation for might as in $(24)^{5}$. The effect is simply to put the content, the quantifiers and the predicates,

5 This interpretation results in readings where modalities have scope over existentials. The interpretation $\llbracket c_{\text {might }}^{\prime} \rrbracket=\lambda v \operatorname{s.} \lambda i k . v \operatorname{si}\left(\lambda i^{\prime} . \diamond\left(k i^{\prime}\right)\right)$ gives existentials having scope over modals. 


$$
\begin{aligned}
\llbracket c_{\text {enter }} \rrbracket & =\lambda s . s(\lambda x i k .(\text { enter } x) \wedge(k i)) & \llbracket c_{\text {wolf }} \rrbracket & =\lambda x i k .(\text { wolf } x) \wedge(k i) \\
\llbracket c_{\text {growl }} \rrbracket & =\lambda s . s(\lambda x i k .(\text { growl } x) \wedge(k i)) & \llbracket c_{i t} \rrbracket & =\lambda P i k . P(\operatorname{sel} i) i k \\
\llbracket c_{a} \rrbracket & =\lambda P Q . \lambda i k . \exists x . P x(x:: i)\left(\lambda i^{\prime} \cdot Q x i^{\prime} k\right) & &
\end{aligned}
$$

Table 1 Basic dynamic lexicon

under the scope of the modal operators.

$$
\llbracket c_{\text {might }} \rrbracket=\lambda v s . \lambda i k . \diamond(v s i k) \quad \llbracket c_{\text {would }} \rrbracket=\lambda v s . \lambda i k . \square(v s i k)
$$

Then, with $t_{0}=c_{\text {might }} c_{\text {enter }}\left(c_{a} c_{\text {wolf }}\right)$ and $t_{1}=c_{\text {would }} c_{\text {growl }} c_{i t} t_{0} \cdot t_{1}$ represents (17) and we get:

$$
\begin{aligned}
\llbracket t_{0} \rrbracket= & \lambda i k \cdot \diamond(\exists x \cdot(\text { wolf } x) \wedge((\text { enter } x) \wedge(k(x:: i)))) \\
\llbracket t_{1} \rrbracket=\lambda i k . \square((\text { growl }(\operatorname{sel} i)) \wedge(k i)) & \\
\llbracket t_{0} \cdot t_{1} \rrbracket=\lambda i k . \diamond & (\exists x .(\text { wolf } x) \wedge((\text { enter } x) \\
& \wedge(\square((\text { growl }(\operatorname{sel}(x:: i))) \wedge(k(x:: i))))))
\end{aligned}
$$

Our lexical entries also have the effect of embedding one modality within the other, making discourse referents (here the wolf $x$ ) accessible for further modal continuation such as it would growl. The first sentence makes this discourse referent explicitly available in the environment $(x:: i)$ of the next sentences.

This is not completely satisfactory because it entails that from a world where a wolf $x$ enters the room, all the accessible worlds verify that $x$ is growling. But $x$ might be a tiger in one of these worlds; such worlds should not affect the evaluation of the would claim. Below we introduce a modal base that restricts the epistemically relevant worlds in the right way.

\subsubsection{Introducing the modal base}

In section 3.1, we specified the atomic type $\gamma$ of the context as a theory, and in section 2 , we made $\gamma$ a list structure, with the attendant update function $::: e \rightarrow \gamma \rightarrow \gamma$ and access function sel $: \gamma \rightarrow e$. We now combine these two ideas by considering $\gamma$ as a record:

$$
\gamma=\left\{\mathrm{m}_{-} \text {ref }: \gamma^{\prime} ; \text { base }: t\right\}
$$

where $\gamma^{\prime}$ provides a set of entities while the base field contains the current modal base. 
A Montagovian treatment of modal subordination

We overload the following functions using the previously defined ones:

$$
\begin{aligned}
x::_{b} i & =\left\{\mathrm{m}_{-} \text {ref }=x::\left(i . \mathrm{m}_{-} \text {ref }\right) ; \text { base }=i . \text { base }\right\} \\
\mathrm{sel}_{b} i & =\operatorname{sel}\left(i . \mathrm{m}_{-} \text {ref }\right) \\
p \wedge_{b} i & =\left\{\mathrm{m}_{-} \text {ref }=\left(i . \mathrm{m}_{-} \text {ref }\right) ; \text { base }=p \wedge(i . \text { base })\right\}
\end{aligned}
$$

and get the lexicon of Table 2. Neither the definition of the $\Omega$ type nor the sentence combination rule change. But standard lexical entries now can add to the modal base (the $k$ continuations are fed with $\left(\right.$ pred $\left.\wedge_{b} i\right)$ parameters) and the lexical entry for might explicitly uses the current modal base $i$.base. The main operator for computing the new modal base is conjunction. However, nothing prevents us from having more complex computations such as various kinds of inference in order to take into account Roberts's analysis of counterfactuals, which exploits consistency relations.

$$
\begin{aligned}
\llbracket c_{\text {enter }} \rrbracket & =\lambda s . s\left(\lambda x i k \cdot(\text { enter } x) \wedge\left(k\left((\text { enter } x) \wedge_{b} i\right)\right)\right) \\
\llbracket c_{\text {growl }} \rrbracket & =\lambda s . s\left(\lambda x i k \cdot(\text { growl } x) \wedge\left(k\left((\text { growl } x) \wedge_{b} i\right)\right)\right) \\
\llbracket c_{a} \rrbracket & =\lambda P Q . \lambda i k . \exists x . P x(x:: i)\left(\lambda i^{\prime} \cdot Q x i^{\prime} k\right) \\
\llbracket c_{\text {wolf }} \rrbracket & =\lambda x i k \cdot(\text { wolf } x) \wedge\left(k\left((\text { wolf } x) \wedge_{b} i\right)\right) \\
\llbracket c_{i t} \rrbracket & =\lambda P i k . P\left(\operatorname{sel}_{b} i\right) i k \quad \llbracket c_{\text {might }} \rrbracket=\lambda v s . \lambda i k . \diamond(i . \text { base } \Rightarrow v s i k)
\end{aligned}
$$

Table 2 Modal context and modal base

Our lexical entries ${ }^{6}$ together with our binder rule in (6) provide the following derivation for the basic example of $\mathrm{MS}^{7}$ :

$$
\begin{aligned}
& \llbracket c_{\text {might }} c_{\text {enter }} \rrbracket=\lambda s . \lambda i k . \diamond\left(i . \text { base } \Rightarrow s\left(\lambda x i k .(\operatorname{enter} x) \wedge\left(k\left((\operatorname{enter} x) \wedge_{b} i\right)\right)\right)\right) \\
& \llbracket c_{a} c_{\text {wolf }} \rrbracket=\lambda Q i k . \exists x \text {. }(\text { wolf } x) \wedge\left(Q x(\text { wolf } x) \wedge_{b}\left(x::_{b} i\right)\right) k \\
& \llbracket t_{0} \rrbracket=\lambda i k . \diamond(i \text {.base } \Rightarrow \exists x \text {. }(\text { wolf } x) \wedge((\operatorname{enter} x) \\
& \left.\left.\wedge\left(k\left((\text { enter } x) \wedge_{b}(\text { wolf } x) \wedge_{b}\left(x::_{b} i\right)\right)\right)\right)\right) \\
& \llbracket t_{1} \rrbracket=\lambda i k . \square\left(i \text {.base } \Rightarrow\left(\operatorname{growl}\left(\operatorname{sel}_{b} i\right)\right) \wedge\left(k\left(\left(\operatorname{growl}\left(\operatorname{sel}_{b} i\right)\right) \wedge_{b} i\right)\right)\right)
\end{aligned}
$$

6 A better interpretation for $c_{i t}$ would be $\lambda P i k$.let $x=\mathrm{sel}_{b} i$ in Pxio to ensure that the selected element remains the same in the modal base and in the modal claim, as the derivations below show. For simplicity, we don't do this here but we assume that the same element is selected in both places without loss of generality.

7 We use the facts that $\left(p \wedge_{b}\left(x::_{b} i\right)\right)$.base $=p \wedge i$.base and that $\left(p \wedge_{b}\left(x::_{b} i\right)\right)$. m_ref $=x:: i$.m_ref. 
so that

$$
\begin{aligned}
\llbracket t_{0} \cdot t_{1} \rrbracket & =\lambda i k \cdot(\diamond(i . \text { base } \Rightarrow \exists x .(\text { wolf } x) \wedge((\operatorname{enter} x) \\
& \wedge\left(\square\left(\left((\text { enter } x) \wedge_{b}(\text { wolf } x) \wedge_{b}\left(x::_{b} i\right)\right)\right) \text {.base } \Rightarrow\left(\text { growl }\left(\operatorname{sel}_{b}\left(x:_{b} i\right)\right)\right)\right. \\
& \left.\left.\left.\left.\wedge\left(k\left((\text { growl }(\operatorname{sel}(x:: i . \text {.m_ref }))) \wedge_{b}\left((\text { enter } x) \wedge_{b}(\text { wolf } x) \wedge_{b}\left(x::_{b} i\right)\right)\right)\right)\right)\right)\right)\right)
\end{aligned}
$$

Then with empty $=\left\{\mathrm{m}_{-}\right.$ref $=\mathrm{nil}$; base $\left.=\top\right\}$ and $\mathbb{T}=(\lambda i . \top)$, the empty parameters for starting a discourse, we get

$$
\begin{aligned}
\llbracket t_{0} \cdot t_{1} \rrbracket \text { empty } \mathbb{T}=\diamond & (T \Rightarrow \exists x .(\text { wolf } x) \wedge((\text { enter } x) \\
& \wedge(\square(((\text { enter } x) \wedge(\text { wolf } x)) \Rightarrow(\text { growl }(\operatorname{sel}((x:: \operatorname{nil}))))))))
\end{aligned}
$$

Our lexical entries again yield logical forms in which the would claim is embedded under the might claim, allowing us to link the anaphoric pronoun via binding to its antecedent using a bound variable, in contrast to (Roberts 1989). To make the claims also hold of the relevant possibilities selected by the might sentence, however, we require a condition on our model theoretic structures: we need an alternativeness relation for the epistemic modality, which is at least secondarily reflexive so that the $\square$ claims also hold of the relevant possibilities selected by the might sentence. One might wonder whether the $\square$ needs to be evaluated in the actual world too; we evaluate it only at those worlds picked out by the might claim. To have the effect of the would claim holding of all epistemic possibilities, we must stipulate the alternativeness relation to be Euclidean.

$\mathrm{CS}$ is flexible enough to allow other lexical entries inducing a wide scope reading for the existential over the modalities and with the modalities unembedded. This permits again a bound variable analysis of the anaphoric link across might and would claims. CS allows us to discuss various interpretations without changing the theoretical framework: we interpret formulas in the standard model theory of modal logic; it is then a matter of lexical semantics to achieve the desired effects.

\subsubsection{Interactions between actual and modal contexts}

To integrate interactions between factual and modal information, we extend our records, our representations of the left context in the previous section, to include the factual information within the left context. We do this by adding a new field f_ref to the record that will contain discourse referents introduced in the factual world:

$$
\gamma=\left\{\mathrm{m}_{-} \text {ref }: \gamma^{\prime} ; \text { base }: t ; \text { f_ref }: \gamma^{\prime}\right\}
$$

Actually, we need this type to be a little bit more complex. It must contain another field encoding the current mode of the sentence so that indefinites will be able to add 
A Montagovian treatment of modal subordination

the introduced variables in $\mathrm{m}_{-}$ref when in a modal context $\left(\left\{\mathrm{m}_{-}\right.\right.$ref $=x:: i . \mathrm{m} \_$ref; $\mathrm{f} \_$ref $=$ $i . f_{-}$ref $\left.\}\right)$and in $\mathrm{f}_{-}$ref when in the actual context $\left(\left\{\mathrm{m}_{-}\right.\right.$ref $=i . \mathrm{m}_{-}$ref; $\mathrm{f}$ _ref $=x:: i$.f_ref $\left.\}\right)$. The same holds for the pronoun that has to select either from the factually introduced referents (sel $i$.f_ref) when in the factual mood or from the union of the factually and modally introduced referents ( $\mathrm{sel} i$.m_ref $\cup i$.f_ref) when in a modal mood. To avoid expressing all the cases within our lexical semantics, we consider a modal entry $a^{m}$ and a factual entry $a^{f}$ for the indefinite $a$, a modal entry $i t^{m}$ and a factual entry $i t^{f}$ for the pronoun, etc.

Our CS now will use two continuations: one containing facts about the actual world, one containing facts about live possibilities the discourse describes. Instead of producing at the end the $t$ type, sentences now produce a pair of claims: one for the epistemic worlds, one for the factual world. We model the pair with the higher-order type function of the type $(t \rightarrow t \rightarrow t) \rightarrow t$. It is important that the result itself is of type $t$ and not of type $(t, t)$, because otherwise quantifiers would be introduced either in the first projection or in the second projection and hence bound variables would never be accessible to both components. But the variables introduced by factual DPs have to be accessible to both components.

These changes induce a change in type for $\Omega$ :

$$
\llbracket s \rrbracket=\gamma \rightarrow(\gamma \rightarrow t) \rightarrow(\gamma \rightarrow t) \rightarrow(t \rightarrow t \rightarrow t) \rightarrow t \triangleq \Omega
$$

$$
\begin{aligned}
& \llbracket c_{\text {enter }} \rrbracket=\lambda s . s\left(\lambda x i k_{1} k_{2} f . f\left((\operatorname{enter} x) \wedge\left(k_{1}\left(\operatorname{enter} x \wedge_{b} i\right)\right)\right)\left((\operatorname{enter} x) \wedge\left(k_{2} i\right)\right)\right) \\
& \llbracket c_{\text {wolf }} \rrbracket=\lambda x i k_{1} k_{2} f . f\left((\text { wolf } x) \wedge\left(k_{1}\left(\text { wolf } x \wedge_{b} i\right)\right)\right)\left((\text { wolf } x) \wedge\left(k_{2} i\right)\right) \\
& \llbracket c_{a}^{m} \rrbracket=\lambda P Q . \lambda i k_{1} k_{2} f . f\left[\exists x . P x\left\{i \text { with m_ref }=x:: i . \mathrm{m}_{-} \text {ref }\right\}\right. \\
& \left.\left(\lambda i^{\prime} \cdot Q x i^{\prime} k_{1} k_{2} \Pi_{1}\right) k_{2} \Pi_{1}\right]\left(k_{2} i\right) \\
& \llbracket c_{a}^{f} \rrbracket=\lambda P Q . \lambda i k_{1} k_{2} f . \exists x . f\left[k_{1}\{i \text { with f_ref }=x:: i\}\right] \\
& {\left[P x\{i \text { with f_ref }=x:: i\} k_{1}\left(\lambda i^{\prime} \cdot Q x i^{\prime} k_{1} k_{2} \Pi_{2}\right) \Pi_{2}\right]} \\
& \llbracket c_{i t}^{m} \rrbracket=\lambda P i k_{1} k_{2} f . P\left(\mathrm{sel}_{b} i . \mathrm{m}_{-} \text {ref } \cup i . \mathrm{f} \_ \text {ref }\right) i k_{1} k_{2} f \\
& \llbracket c_{i t}^{f} \rrbracket=\lambda P i k_{1} k_{2} f . P\left(\mathrm{sel}_{b} i . \text { f_ref }\right) i k_{1} k_{2} f \\
& \llbracket c_{\text {might }} \rrbracket=\lambda v s . \lambda i k_{1} k_{2} f . f\left(\diamond\left(i \text {. base } \Rightarrow\left(v s i k_{1} k_{2} \Pi_{1}\right)\right)\right)\left(k_{2} i\right)
\end{aligned}
$$

Table 3 Modal and factual contexts 
Together with the lexical entries of Table $3^{8}$, we get (with $t_{2}=c_{\text {will }} c_{\text {growl }} c_{i t}$ ):

$$
\begin{aligned}
& \llbracket t_{0} \rrbracket=\lambda i k_{1} k_{2} f . f[\diamond(i \text {.base } \Rightarrow \exists x .(\text { wolf } x) \wedge((\text { enter } x) \\
& \left.\left.\left.\wedge\left(k_{1}\{i \text { with m_ref }=x:: i \text {.m_ref } ; \text { base }=(\text { wolf } x) \wedge(\text { enter } x) \wedge i \text {.base }\}\right)\right)\right)\right] \\
& {\left[k_{2} i\right]}
\end{aligned}
$$

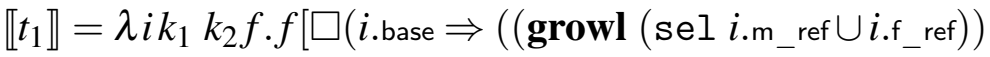

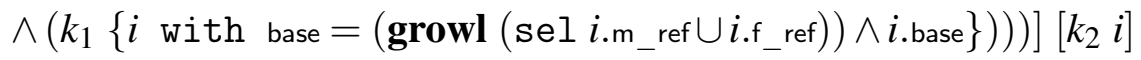

$$
\begin{aligned}
& \llbracket t_{2} \rrbracket=\lambda i k_{1} k_{2} f . f\left[k_{1} i\right]\left[\left(\text { growl }\left(\text { sel } i . f_{-} \text {ref }\right)\right) \wedge\left(k_{2} i\right)\right]
\end{aligned}
$$

To combine our more complex logical forms, we require a new binder rule:

$$
\llbracket S_{1} \cdot S_{2} \rrbracket=\lambda i k_{1} k_{2} f \cdot \llbracket S_{1} \rrbracket i\left(\lambda i^{\prime} \cdot \llbracket S_{2} \rrbracket i^{\prime} k_{1} k_{2} \Pi_{1}\right)\left(\lambda i^{\prime} \cdot \llbracket S_{2} \rrbracket i^{\prime} k_{1} k_{2} \Pi_{2}\right) f
$$

To simplify, we interpret our examples with empty environments (empty = $\left\{\mathrm{m}_{-}\right.$ref $=\mathrm{nil}$; base $=\top$; f_ref $\left.\left.=\mathrm{nil}\right\}\right)$, trivial continuations $(\mathbb{T}=\lambda i . \top)$. Conjunction of the two components (Conj $=\lambda b_{1} b_{2} . b_{1} \wedge b_{2}$ ) yields the type $t$. This yields:

$$
\begin{aligned}
\llbracket t_{0} \cdot t_{1} \rrbracket \text { empty } \mathbb{T} \mathbb{T} \text { Conj }=[\diamond & (\top \Rightarrow(\exists x .(\text { wolf } x) \wedge((\text { enter } x) \\
\wedge & (\square(((\text { wolf } x) \wedge(\text { enter } x)) \\
& \Rightarrow(\text { growl }(\text { sel }((x:: \text { nil }) \cup \text { nil } 1)))))))] \wedge \top \\
\llbracket t_{0} \cdot t_{2} \rrbracket \text { empty } \mathbb{T} \mathbb{T} \text { Conj }=[\diamond & (\top \Rightarrow(\exists x .(\text { wolf } x) \wedge((\text { enter } x) \wedge \top)))] \\
& \wedge[\text { growl }(\text { sel nil })]
\end{aligned}
$$

In the first case, the sel function has access to $x$, so (17), represented by $t_{0} . t_{1}$, is predicted to be OK, but (18), represented by $t_{0} \cdot t_{2}$, is predicted to be bad, because $x$ is not part of the accessible environment. To illustrate the importance of pronouns within modal environments selecting from both modal and factual contexts, consider again (19).

A wolf is outside. He might eat you.

The first indicative sentence yields upon $\beta$ reduction:

$$
\begin{aligned}
& \lambda i k_{1} k_{2} f . \exists x . f\left[k_{1}\left\{i \text { with f_ref }=x:: i_{\text {.f_ref }}\right\}\right][(\text { wolf } x) \wedge((\text { Outside } x) \wedge \\
& \left.\left.\left(k_{2}\{i \text { with f_ref }=x:: i \text {.f_ref }\}\right)\right)\right]
\end{aligned}
$$

Now integrating the modalized second sentence, we get:

$$
\exists x .[\diamond(\top \Rightarrow(\text { eat you }(\operatorname{sel} \operatorname{nil} \cup(x:: \operatorname{nil}))))] \wedge[(\text { wolf } x) \wedge((\text { Outside } x))]
$$

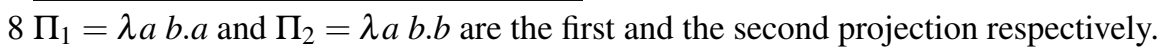


A Montagovian treatment of modal subordination

And this is just what we want.

Exploiting a compositional analysis of counterfactuals (Asher \& McCready 2007), our approach can also treat anaphoric links across counterfactuals: ${ }^{9}$

$$
\text { If I had bought a refrigerator, I wouldn't know what to do with it. }
$$

\subsubsection{Putting everything together}

Modal subordination phenomena of Veltman's observations show that factual information is accessible to modalized continuations. With a slight abuse of notation (to avoid even longer $\lambda$-terms), we make a factual sentence update the world context. This suffices to capture Veltman modalities. ${ }^{10}$

So we now add to the context a field theory to the context that will contain the theory under construction which is tested as in Section 3.1.1 and we have

$$
\gamma \triangleq\left\{\mathrm{m}_{-} \text {ref }: \gamma^{\prime} ; \text { base }: t ; \text { f_ref }: \gamma^{\prime} ; \text { theory }: \text { THEORY }\right\}
$$

Our final lexical entry for might is this:

$$
\begin{aligned}
\llbracket c_{m i g h t} \rrbracket=\lambda v s . \lambda i k_{1} k_{2} f .(\lambda P .(\operatorname{TEST} P) i \text {.theory } \\
\left.\left(\lambda i^{\prime} o_{1}^{\prime} o_{2}^{\prime} f^{\prime} \cdot f^{\prime}\left(\diamond\left(i^{\prime} \text {.base } \Rightarrow\left(P i^{\prime} o_{1}^{\prime} o_{2}^{\prime} P i_{1}\right)\right)\right)\right)\right)(v s) i k_{1} k_{2} f
\end{aligned}
$$

We have now captured our two types of context sensitivity for epistemic modals within a unified framework, revealing the power of the CS approach. We've added Veltman style modalities in conservative fashion to the externally static but internally dynamic modalities needed to capture MS. We've also shown how the choice of lexical entries and binder rules determine features of modal contexts.

\section{Attitude dynamics}

Modal subordination also occurs between pairs of attitude reports involving one or more cognitive agents. Consider:

(38) Sam wants to marry an Italian. He hopes she will be rich.

(39) Hob thinks a witch has blighted his mare, and Nob thinks she has given his cow warts.

9 The present formalism does not suffice to handle examples with negation like

$$
\text { I didn't buy a refrigerator. I wouldn't know what to do with it. }
$$

We follow Asher \& McCready's (2007) explanation in terms of discourse structure. 10 We ignore here some further subtleties, like those explored in Asher \& McCready 2007. 
CS is flexible enough to allow lexical entries inducing a wide scope reading for the existential quantifier over unembedded modalities for MS. The intuitive truth conditions for (38) and (39) require such modal independence. In (38) Sam doesn't want to hope that his Italian is rich-he simply hopes that she will be rich. Similarly, (39) is not intuitively a report about what Hob believes about Nob or vice-versa.

The wide-scope strategy yields the following for (39), given an empty left context and trivial continuation:

$$
\exists x\left(\mathscr{B}_{h}(\text { witch }(x) \wedge \text { blighted ... }(x)) \wedge \mathscr{B}_{n}(\text { gave warts... }(\text { sel }((x:: \operatorname{nil}))))\right)
$$

The truth of (40) problematically requires that there is an object in the world of evaluation that is a witch in all of Hob and Nob's belief worlds. The witch could be entirely a figment of Hob and Nob's imagination. We don't want the truth conditions of such reports to rest on substantive assumptions about the metaphysics of modality.

Asher (1987) provided the first dynamic treatment of such examples. He exploited the contents of DRSs as sets of world assignment pairs (as Frank \& Kamp would do later for modal subordination), and coordinated the interpretations of two attitude descriptions by constraining the proper embeddings of each to agree on assignments to certain pairs of discourse referents. On such an approach, $a$ witch in (39) was treated introducing material only within the scope of the belief operator; various possible witches could be the value of this variable or discourse referent under the coordinated assignments. The Hob-Nob examples, like the MS examples, showed the peculiarities of the logical framework of DRT in which discourse referents have a kind of hybrid status, somewhere between bound and free variables.

To capture Asher's (1987) interpretation of (39) but the curious logical status of the discourse referents in DRT, we propose that the truth conditions of (39) entail Hob and Nob's sharing of an individual concept. Without going into details (though see Asher 2010), the story exploits coercion and our TEST operator of section 3 . The pronoun in the second sentence presupposes the presence of an antecedent of the appropriate type. Like other presuppositional triggers, the pronoun places a TEST on the antecedent context that there be an antecedent of the appropriate type in the left context. Given our interpretive assumptions, this is not the case. Thus, the TEST fails. But the semantics of the pronoun also licenses an accommodation mechanism for the exception, whereupon the antecedent changes its type from $e$ to $s \rightarrow e$, the type of an individual concept. ${ }^{11}$ To treat the exception, we then redo the entire computation having lifted the type of the indefinite to a quantifier over individual concepts.

11 We can imagine the same sort of test mechanism to be triggered not only because the context fails the TEST for semantic reasons, as here, but also for pragmatic reasons, as in the famous paycheck sentences- the man who gave his paycheck to his wife was wiser than the one who gave it to his mistress. 
A Montagovian treatment of modal subordination

To treat the exception case for the TEST properly, we must precisify the particular kind of individual concept at issue. The intuitive truth conditions of (39) dictate that we need to ensure that the extension of the concept in Nob's belief worlds and the extension of the concept in Hob's worlds are such that: if witch $A$ blighted Hob's mare in a Hob belief world, then at least some of the belief worlds of Nob will have witch $A$ giving his cow warts; and all of Nob's belief worlds, anyone who gave Nob's cow warts is a witch who blighted Hob's mare in one of Hob's belief worlds. This is what the coordinated dependencies of Asher 1987 captures. This will require a more complex binder rule making special use of the modal continuation.

TY2, with an added basic type for worlds, proves useful for treating the exception to the TEST. In the language of TY2, roughly all predicates that were $n$-ary become $n+1$-ary with the addition of a world parameter. The exception rule forces the evaluation of the concept in Nob's belief worlds to be the same the extension of the concept in some one of Hob's belief worlds. The end result is this:

$$
\begin{aligned}
\lambda w . & \exists x \rightarrow e \\
& \wedge \exists w^{\prime} \in \mathscr{B}_{h, w}\left(\operatorname{witch}\left(x\left(w^{\prime}\right), w^{\prime}\right) \wedge \text { blighted-horse }\left(x\left(w^{\prime}\right), w^{\prime}\right)\right. \\
& \left.\left.\wedge \forall w^{\prime \prime} \in \mathscr{B}_{n, w}\left(\text { gave-warts }\left(x\left(w_{1}\right)=x\left(w^{\prime}\right), w^{\prime \prime}\right) \wedge \exists w^{\prime \prime \prime} \in \mathscr{B}_{h, w} x\left(w^{\prime \prime \prime}\right)=x\left(w^{\prime \prime}\right)\right)\right)\right)
\end{aligned}
$$

Thus, CS proves very useful in the analysis of dependencies between modalities and between modal and factual information.

\section{References}

Asher, Nicholas. 1987. A typology for attitude verbs and their anaphoric properties. Linguistics and Philosophy 10. 125-198. doi:10.1007/BF00584317.

Asher, Nicholas. 2010. Lexical meaning in context: A web of words. Cambridge University Press.

Asher, Nicholas \& Eric McCready. 2007. Were, would, might and a compositional account of counterfactuals. Journal of Semantics 24(2). 93-129. doi:10.1093/jos/ffl013.

Barker, Chris. 2004. Continuations in natural language. In 4th Continuations Workshop, .

Bernardi, Raffaella \& Michael Moortgat. 2010. Continuation semantics for the lambek-grishin calculus. Information and Computation 208(5). 397-416. doi:10.1016/j.ic.2009.11.005. Special Issue: 14th Workshop on Logic, Language, Information and Computation (WoLLIC 2007).

Brasoeavnu, Adrian. 2008. Structured anaphora to quantifier domains. To appear in Information and Computation. 
Dekker, Paul. 1999. Scopes in discourse. Journal of Language and Computation 1. 7-32.

Fernando, Tim. 1994. What is a DRS? Tech. Rep. R2.1.B Dyana deliverable. Also in the proceedings of a workshop on Computational Semantics (Tilburg, The Netherlands), December 1994.

Frank, Anette. 1996. Context dependence in modal constructions: University of Stuttgart dissertation.

Frank, Anette \& Hans Kamp. 1997. On context dependence in modal constructions. In Semantics and Linguistics Theory 7 (SALT 7), .

Geurts, Bart. 1996. Presupposing: University of Stuttgart dissertation.

Groenendijk, Jeroen \& Martin Stokhof. 1991. Dynamic predicate logic. Linguistics and Philosophy 14(1). 39-100. doi:10.1007/BF00628304.

Groenendijk, Jeroen \& Martin Stokhof. 1996. Changing the context: Dynamics and discourse. In Edit Doron et al (ed.), 11th Annual Conference and of the Workshop on Discourse (IATL 3), Jerusalem: The Israel Association for Theoretical Linguistics.

de Groote, Philippe \& Ekaterina Lebedeva. 2010. Presupposition accomodation as exception handling. In 11th Annual SIGdial Meeting on Discourse and Dialogue (SIGDIAL 2010), .

Janssen, Theo. 1983. Foundations and applications of montague grammar: University of Amsterdam dissertation.

Kamp, Hans. 1981. A theory of truth and semantic representation. In Jeroen A. Groenendijk, Theo Janssen \& Martin Stokhof (eds.), Formal methods in the study of language, Foris.

Moggi, Eugenio. 1991. Notions of computation and monads. Information and Computation 93(1). 55-92. doi:10.1016/0890-5401(91)90052-4.

Philippe de Groote. 2006. Towards a Montagovian account of dynamics. In Semantics and Linguistic Theory 16 (SALT 16), .

Roberts, Craige. 1989. Modal subordination and pronominal anaphora in discourse. Linguistics and Philosophy 12(6). 683-721. doi:10.1007/BF00632602.

Roberts, Craige. 1996. Anaphora in intensional contexts. In Shalom Lappin (ed.), Handbook of contemporary semantic theory, 215-246. Blackwell.

Shan, Chung-chieh \& Chris Barker. 2006. Explaining crossover and superiority as left-to-right evaluation. Linguistics and Philosophy 29(1). 91-134. doi:10.1007/s10988-005-6580-7.

Stone, Matthew \& Daniel Hardt. 1997. Dynamic discourse referents for tense and modals. In International Workshop Proceedings on Computational Semantics 2 (IWCS 2), .

Veltman, Frank. 1996. Defaults in update semantics. Journal of Philosophical Logic 25(3). 221-261. doi:10.1007/BF00248150. 
A Montagovian treatment of modal subordination

Nicholas Asher

IRIT - Université Paul Sabatier

118 route de Narbonne

31062 Toulouse Cedex 4

France

asher@irit.fr
Sylvain Pogodalla

INRIA

Calligramme - Bât. B

615 , rue du Jardin Botanique 54602 Villers-lès-Nancy Cedex

France

Sylvain.Pogodalla@inria.fr 